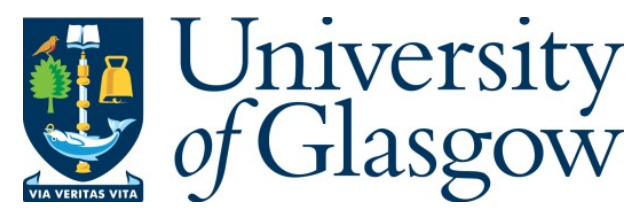

Kallepalli, A. , Innes, J., Gibson, G. M. and Padgett, M. J. (2021) Single-pixel imaging pattern sets and their implications on scene reconstruction. In: Proceedings SPIE 11881, Quantum Technology: Driving Commercialisation of an Enabling Science II (SPIE Photonex), Glasgow, Scotland, United Kingdom, 28 September - 1 October 2021, 18810X.

(doi:10.1117/12.2601228)

This is the Author Accepted Manuscript.

There may be differences between this version and the published version. You are advised to consult the publisher's version if you wish to cite from it.

http://eprints.gla.ac.uk/255548/

Deposited on: 9 November 2021

Enlighten - Research publications by members of the University of Glasgow http://eprints.gla.ac.uk 


\title{
Single-pixel imaging pattern sets and their implications on scene reconstruction
}

\author{
Akhil Kallepalli ${ }^{\mathrm{a},}{ }^{,}$, John Innes ${ }^{\mathrm{b}}$, Graham M. Gibson ${ }^{\mathrm{a}}$, and Miles J. Padgett ${ }^{\mathrm{a}}$

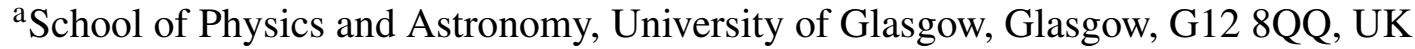 \\ b'Innovation \& Technology Group, Leonardo MW Ltd., Edinburgh, EH3 9GL, UK
}

\begin{abstract}
Alternatives to focal-plane detector arrays have emerged in recent years and are now being widely investigated. One of the most promising of these are single-pixel imaging techniques. Single-pixel techniques recreate a scene using the knowledge of projected patterns and the measured backscattered signals. This research investigates the implications of using idealised patterns, Fourier-transformed patterns and camera-captured patterns generated by plane wave decomposition methods. Hadamard patterns are projected into the far-field of the phased-array modulator source and used for robust reconstruction in a reflective arrangement. The choice of the optimal pattern sets from these sources are used for single-pixel imaging reconstruction and compressed sensing. The technique is robust to poor signal-to-noise conditions and is applicable in cases where a limited number of measurements are possible. Our technique and methodology can be further applied to any region of the electromagnetic spectrum where phased-array sources are available, such as in the radar regime.
\end{abstract}

Keywords: Spatial light modulators, compressed sensing, ghost imaging, phase modulation, Hadamard matrices

\section{INTRODUCTION}

Traditional digital imaging systems use a lens to form an image of the scene on a focal-plane detector array, with each element of the array recording the corresponding intensity of the image pixel. Single-pixel imaging systems are an alternative to this, where the detector array is replaced by a spatial light modulator (SLM) which applies a mask to the image and then a large-area, single-pixel detector subsequently measures the total optical power transmitted by this mask. ${ }^{1}$ This measured power is proportional to the overlap integral between the imaged scene and the chosen mask. By measuring the overlap integral for a wide range of different masks, it is possible, using various algorithms, to infer the image of the scene. These single-pixel approaches are useful in scenarios where focal-plane detector arrays are expensive due to their operating wavelength. The technique is also applicable in situations where the enhanced time resolution of the single-pixel detector gives other detection modalities ${ }^{2}$ such as depth-resolved imaging, ${ }^{3-5}$ microscopy, ${ }^{6,7}$ Raman imaging,,${ }^{8,9}$ to name a few. The choice of masks and the reconstruction algorithm are also factors when implementing compressed sensing, ${ }^{10-12}$ wherein a high-quality image is reconstructed using fewer measurements than in traditional approaches.

Single-pixel imaging systems can be configured in two distinct ways (Figure 1). In one approach, the object is illuminated uniformly and the backscattered light is imaged onto an SLM. A mask is applied to the light at the SLM and the resulting filtered light is measured (in transmission or reflection modes). In the other approach, the mask is applied to the light prior to interaction with the object. The modulated light interacts with the object and the resulting reflected/transmitted light is measured. The latter approach is comparable to computational ghost imaging. ${ }^{14}$ Clearly, the choice of mask design and reconstruction algorithms are significant and have the same considerations.

A commonly chosen basis for masks are Hadamard matrices due to their orthogonality. ${ }^{15-17}$ In this research, a spatial light modulator based on a liquid crystal layer on a silicon backplane (LC-SLM) is used to create spatially varying phase masks. LC-SLMs are capable of creating 128 different phase delays, in the 0-2 $\pi$ range, for each effective pixel of the modulator. Although such a phase modulator can be combined with other optics to create a intensity modulator, the phase-only nature of the LC-SLM itself creates unique opportunities for modulating the light. In addition to phase-only modulation, the LC-SLM can also incorporate diffractive optical elements, which allows the creation of computer generated holograms and intensity distributions in the far-field. When generating the spatial form of the required diffractive pattern, we use the

For additional information regarding the research and the open-source dataset, please send all correspondence to

A.K. (Akhil.Kallepalli@glasgow.ac.uk) or M.J.P. (Miles.Padgett@glasgow.ac.uk) 

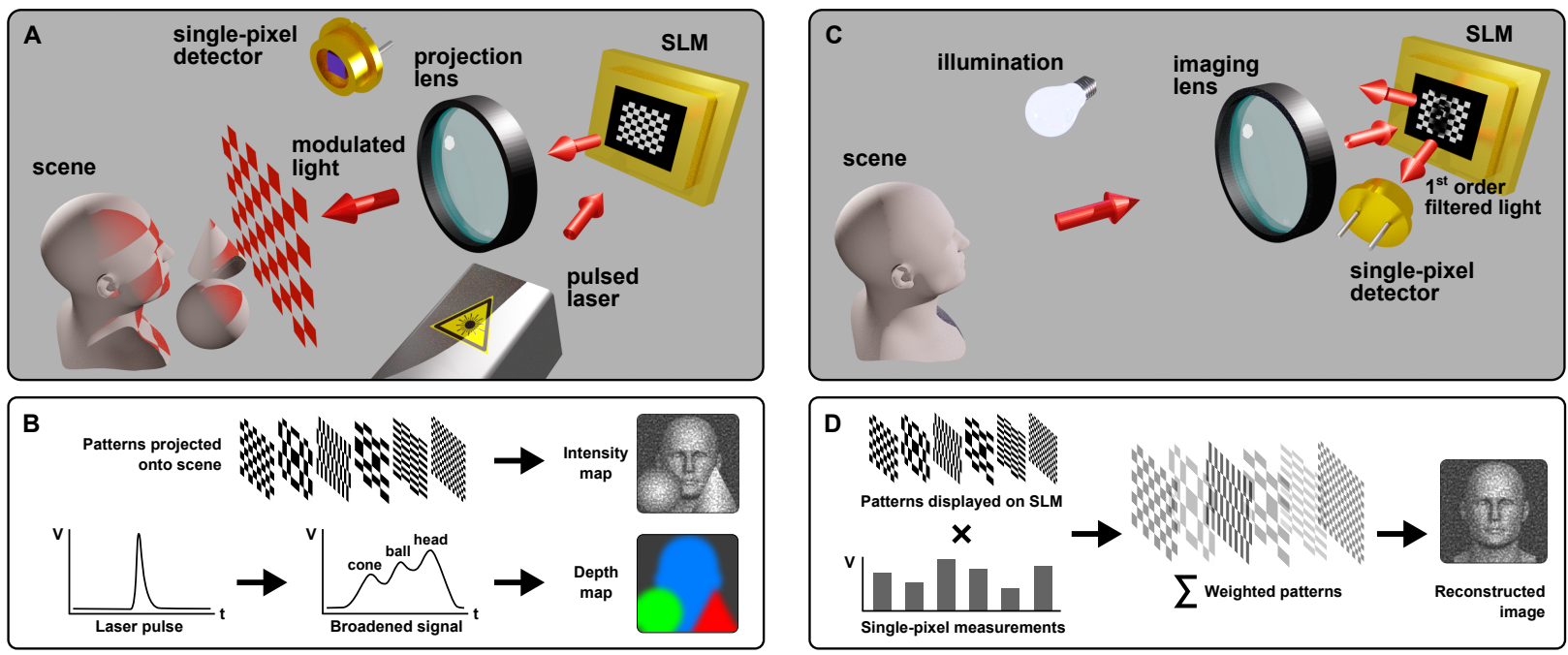

Figure 1. Single-pixel imaging can be achieved in two arrangements using modulated light (A, B) or flood illumination (C, D). The most important and common elements in both arrangements is the spatial light modulator (SLM) and the choice of patterns. Author figure reproduced from Gibson, Johnson and Padgett (2020). ${ }^{2}$

Gerchberg-Saxton (GS) algorithm. ${ }^{18}$ We assess the suitability of the Hadamard pattern sources (idealised/hologram kinoform/imaged intensity) for those that contribute the least in terms of noise and/or uncertainty. The ideal patterns calculated by the algorithm provide the least amount of noise for reconstruction, while the imaged patterns inherently carry the noise induced by the optical elements in the experimental arrangement (Figure 3). Although the patterns from any of these three sources allows reconstruction, a key contribution of this research is an illustration of the capability to reconstruct an image of the scene from both a single-pixel detector (PMT) and an imaging camera (sCMOS).

In this work, spatial phase distribution patterns are calculated using the Gerchberg-Saxton algorithm and displayed on the SLM. The intensity patterns are propagated to the far-field of the SLM to illuminate the target. The power backscattered by the object is measured using a photomultiplier tube (PMT) acting as a single-pixel detector. The knowledge of the mask patterns and the measured power is used to reconstruct an image of the scene. In addition, we also successfully illustrate compressed sensing with a selected subset of the pattern set to reconstruct the image, gaining the advantage of faster imaging and/or robustness in low signal-to-noise ratio (SNR) conditions. We would like to highlight, at this stage, that the methodology presented in this study (in the optical regime) can be applied to any domain that uses phased array sources, such as in the radar regime.

\section{METHODS AND RESULTS}

As illustrated in figure 2, the monochromatic light at $633 \mathrm{~nm}$ from the He-Ne laser fills the aperture of the spatial light modulator (SLM). The SLM is programmed to modulate the beam with the necessary diffractive optic kinoform, as calculated using the Gerchberg-Saxton algorithm, ${ }^{18}$ to create the Hadamard patterns in the far-field plane. The GS algorithm was developed as an iterative solution for spatial phase structure in cases where the intensity in the diffraction and imaging planes are both defined. Using the relations devised by Gerchberg and Saxton (1971), ${ }^{18}$ the phase mask for the SLM is calculated and superposed onto the incident beam. The idealised Hadamard patterns are known at this stage. Subsequently, once encoded on the SLM, the hologram is propagated by fast Fourier transformation and plane wave decomposition to simulate the propagation through the optical system (as detailed in Figure 2) to be used for image reconstruction. The hologram kinoform is recorded after this step. Finally, the patterns propagated and imaged at the sCMOS sensor are also measured and recorded for image reconstruction.

Of these sources, figure 3 shows instances of the ideal Hadamard pattern and the corresponding, real far-field intensity distribution as imaged by the sCMOS camera for comparison. Clearly, laser illumination results in speckle noise in the far-field as seen in the images captured by the sCMOS camera. When the images from the camera that have speckle noise are used in the reconstruction, the final reconstruction is visibly poorer due to the noise added to the signal by 


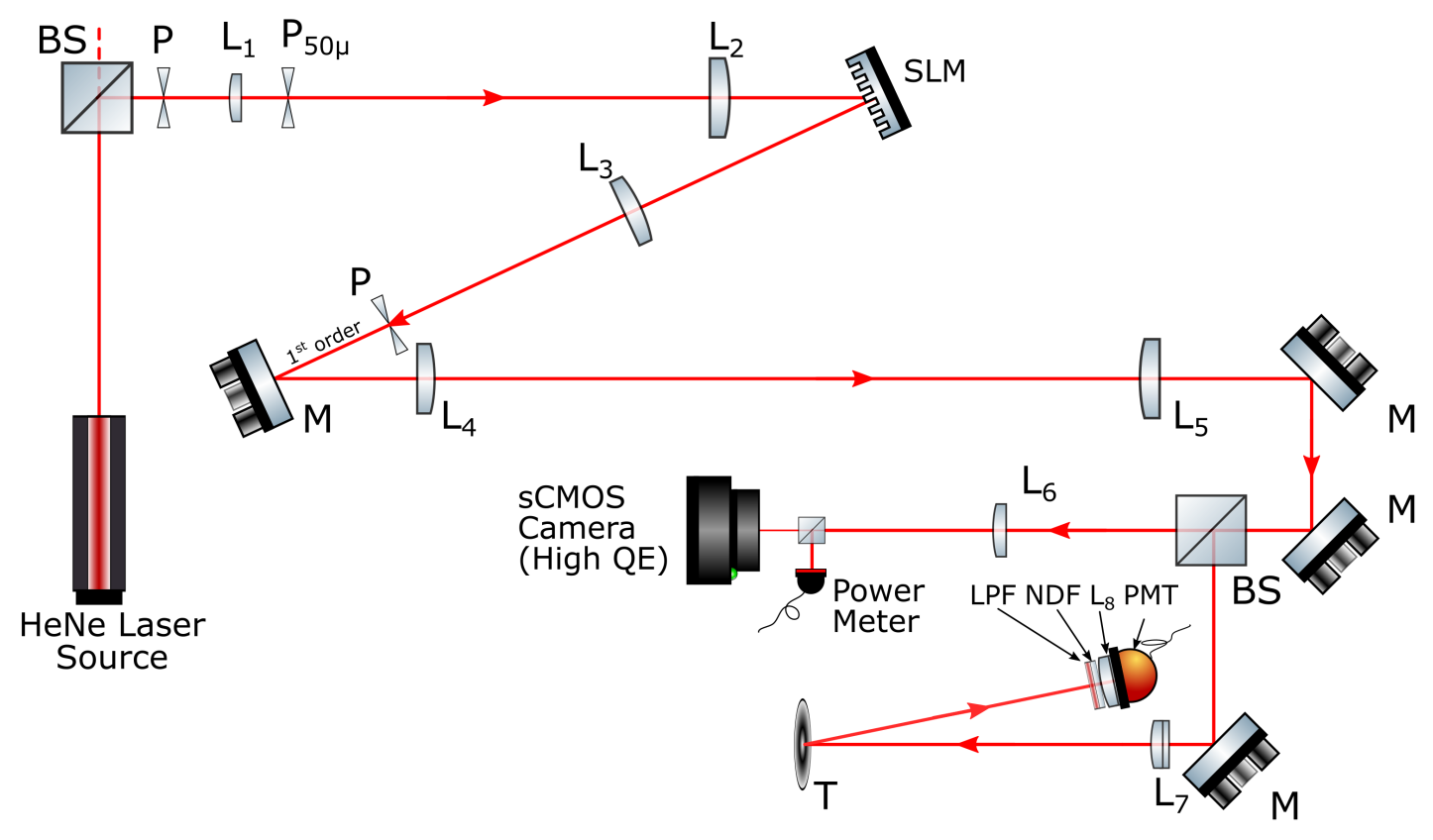

Figure 2. Monochromatic light at $633 \mathrm{~nm}$ is spatially filtered and expanded to fill the aperture of a spatial light modulator (SLM), using a combination of aperture $(\mathrm{P}), 50 \mathrm{~mm}$ focal length lens $\left(\mathrm{L}_{1}\right), 50 \mu \mathrm{m}$ precision pinhole $\left(\mathrm{P}_{50 \mu}\right)$ and $400 \mathrm{~mm}$ focal length lens $\left(\mathrm{L}_{2}\right)$. This light is modulated using diffraction grating patterns displayed on the SLM. The first order diffracted light is selected using a $250 \mathrm{~mm}$ focal length lens $\left(\mathrm{L}_{3}\right)$ and aperture $(\mathrm{P})$. This light is subsequently propagated into the far-field through a $250 \mathrm{~mm}$ focal length lens $\left(\mathrm{L}_{4}\right)$ and a $400 \mathrm{~mm}$ focal length lens $\left(\mathrm{L}_{5}\right)$. The beam is propagated into two separate arms using beamsplitter (BS). One arm uses a power meter to measure the laser power and an ORCA-Flash CMOS Hamamatsu camera that images the illumination pattern. The second arm consists of the target (T) of which light is reflected towards an arrangement of a long pass filter (LPF), neutral density filters (NDF), an $80 \mathrm{~mm}$ focal length lens $\left(\mathrm{L}_{8}\right)$ and a Thorlabs photomultiplier tube (PMT, model PMM02), completing the single-pixel system. Author figure reproduced from Kallepalli, Innes and Padgett $(2021)^{13}$

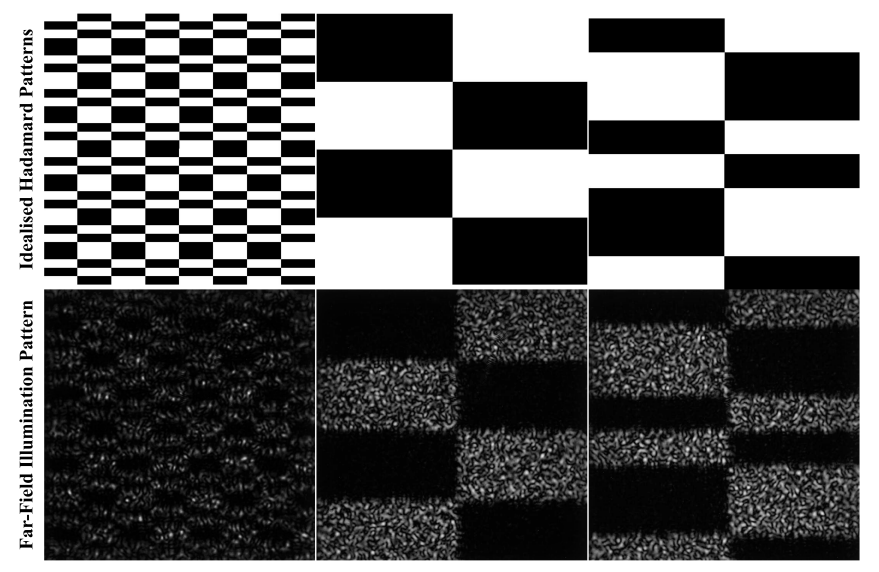

Figure 3. The Hadamard patterns (idealised) are projected in the far-field, and captured using a sCMOS camera (ref. figure 2) to illustrate the illumination distribution on the target. In addition to these pattern sets, the Hadamard matrices are also Fourier-transformed to generate a modelled set of patterns. Author figure reproduced from Kallepalli, Innes and Padgett (2021) ${ }^{13}$

the optical components and the sensor itself, in addition to speckle noise. Although each Gerchberg-Saxton derivation of our kinoform is seeded with a different random start to mitigate speckle noises (on average), the overall contribution to a poorer reconstruction is still significant. Finally, due to the integration time needed to use the camera system, the overall reconstruction is also much slower due to the computational load from a large sensor. Although all three pattern measurements schemes were tested, the choice is motivated by the least contribution of noise; which is in the case of the idealised patterns. As a consequence of this, the imaged intensity patterns and the Fourier-transformed patterns were not 


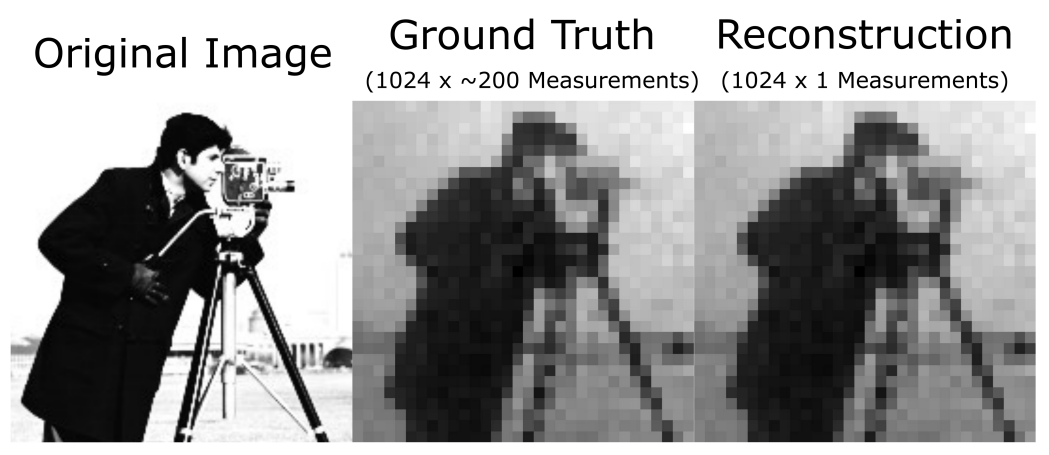

$1024 \times 2$ Measurements $1024 \times 3$ Measurements $1024 \times 4$ Measurements $1024 \times 5$ Measurements

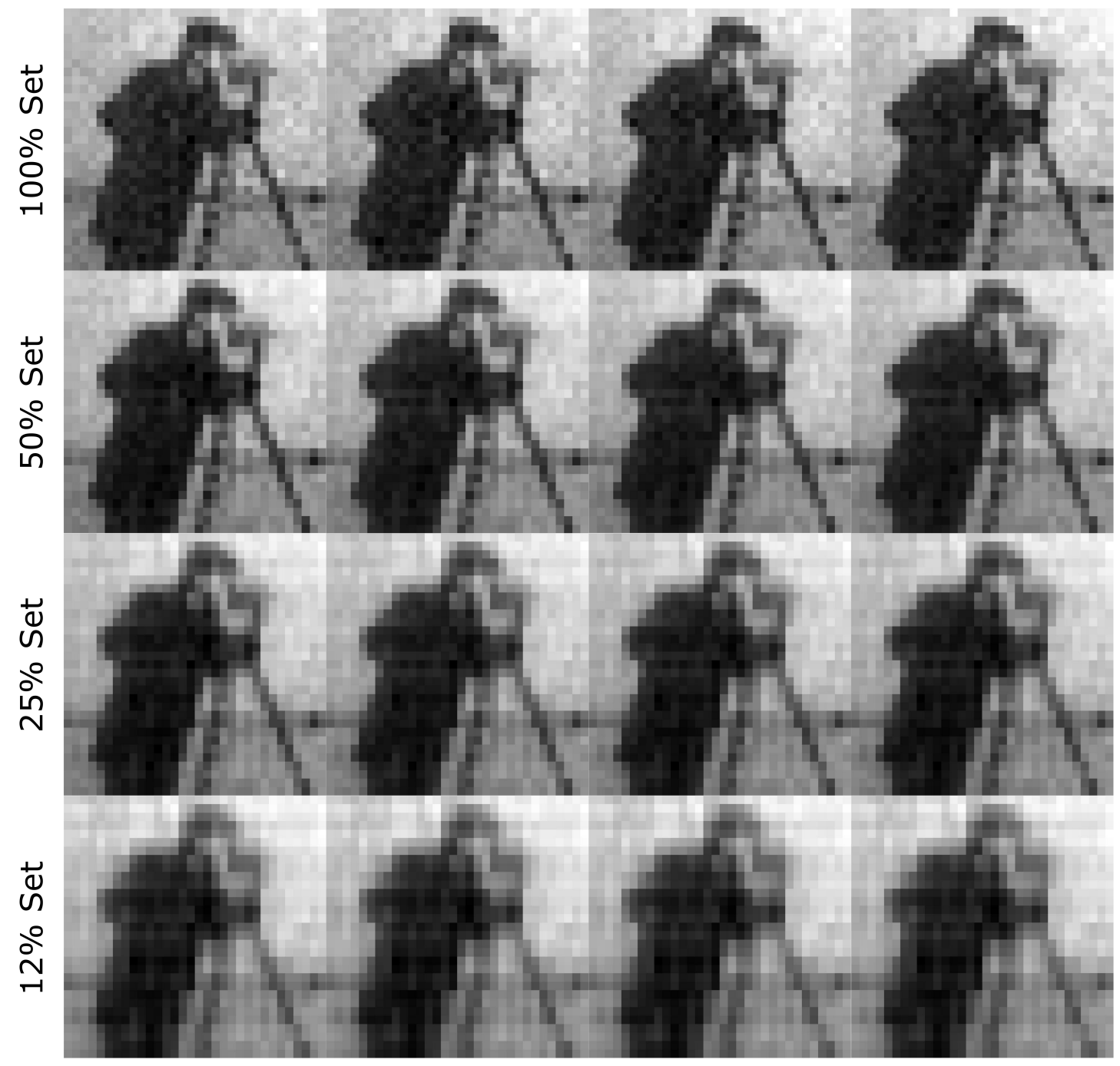

Figure 4. This illustration of compressed sensing uses the standard cameraman test image as an object in the experiment. The ground truth image obtained after $\approx 200$ iterations of 1024 patterns is compared to reconstruction using $100 \%, 50 \%, 25 \%$ and $12 \%$ pattern subsets for up to 5120 measurements. The images here were acquired in a high SNR conditions corresponding to Figure 5 (A). The cameraman image is adapted from the Massachusetts Institute of Technology, all rights reserved. Author figure reproduced from Kallepalli, Innes and Padgett $(2021)^{13}$ 

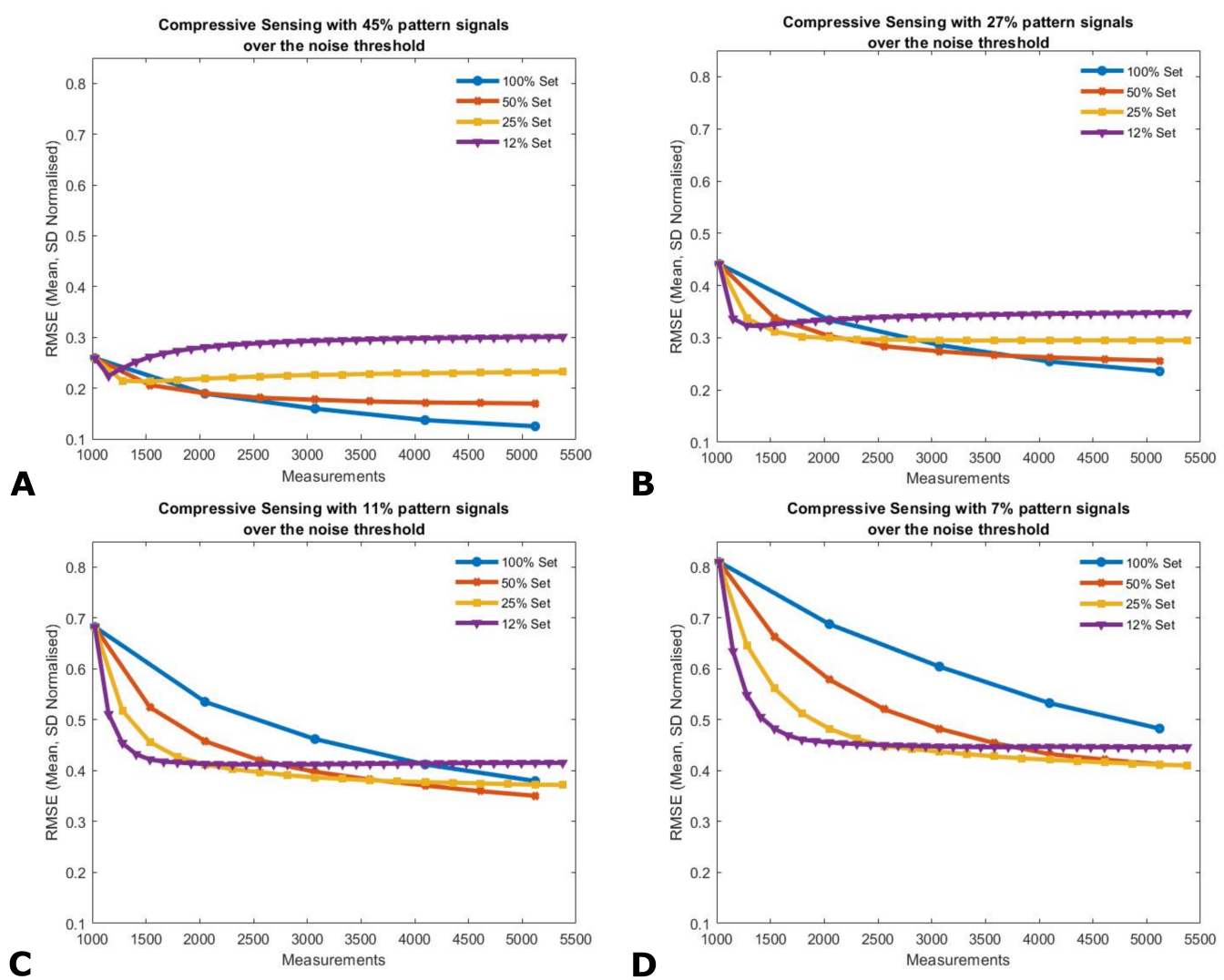

B

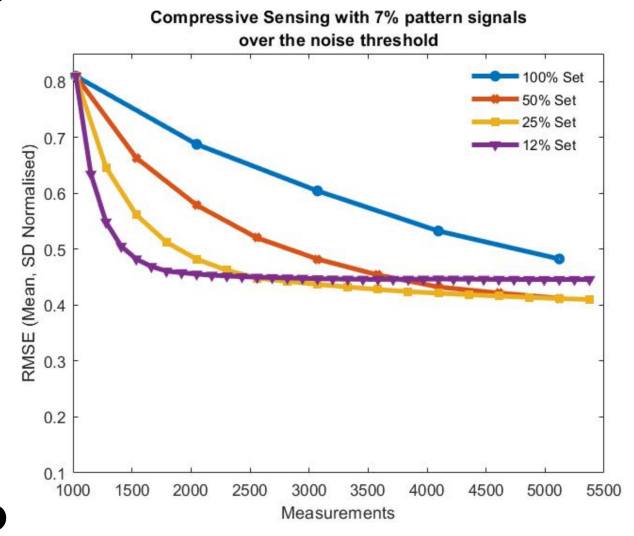

Figure 5. RMS errors of image reconstruction outputs are calculated with respect to the ground truth image as a function of the number of measurements for varying degrees of image compression and SNR regimes. The cases presented here are the ideal condition (A) and the three subsequent scenarios (B-D) with increasingly poor SNR. In low SNR conditions (C, D), compressed sensing shows a better reconstruction up until 3000-4000 patterns. However, the overall image quality does reduce as a function of SNR. Author figure reproduced from Kallepalli, Innes and Padgett $(2021)^{13}$

used in the final image reconstruction. This comes with a significant computational advantage and therefore, a quicker reconstruction.

A phase-grating is added to the kinoform to shift the pattern to the first-order where it is selected with an aperture. The chosen order is propagated through the optical arrangement and into two paths - one with an sCMOS camera and the other with a single-pixel detector (photomultiplier tube, PMT). The PMT is positioned to collect the light backscattered from the object. We measured the corresponding signal acquired by the photomultiplier tube (PMT) when the object was illuminated by a sequence of $i=1 \ldots 1024$ patterns (corresponding to a full set of 32-by-32 patterns). If the Hadamard patterns are described as $p_{i}(x, y)$ and the corresponding signals detected are $s_{i}$, then using traditional reconstruction algorithms (Eq. 1), we can reconstruct the image $I$ from 1024 patterns: ${ }^{19}$

$$
I=\sum_{i=1}^{N}\left(s_{i}-\left\langle s_{i}\right\rangle\right) p_{i}(x, y)
$$

where $\left\langle s_{i}\right\rangle$ is the average value of all single-pixel detector signals.

As previously mentioned, we initially considered image reconstruction using the PMT signals and (1) the ideal Hadamard patterns, (2) calculated holograms (which were generated using the iterative GS calculations and then propagated by planewave decomposition to the object plane) and (3) intensity patterns imaged by the sCMOS camera. Initial testing showed a better image reconstruction and contrast using the measured signals and the idealised patterns, and therefore, became the choice for all subsequent image reconstruction.

In ideal conditions, with unlimited resources and time, the reconstruction of the scene/object is done using numerous iterations of the full pattern set to average the individual images and overcome noise. We established a "ground truth" 
using this approach by averaging $\approx 200$ iterations of the full pattern set. However, in practical situations, the common constraints are higher noise levels and limitations on the number of measurements that can be obtained within a given time. In such cases, compressed sensing, which utilises a subset of patterns and measurements, is an option. Our approach, in comparison to previously presented methods, ${ }^{20-22}$ uses a single PMT for signal measurement and remains robust in poor SNR conditions. The subset of the patterns used are chosen based on their contribution to the image reconstruction, quantified by the corresponding measured signal strength. This approach has no reliance on image libraries or image priors, and therefore can be applied to arbitrary scenes.

With the constraint of a fixed number of measurements, the reconstruction focuses on utilising measurement of the subset of patterns that contribute most significantly. The image acquisition is initiated by a full set of patterns (1024 in the first iteration) after which the measured signals are chosen based on the signal strength and a predefined choice set. Once the subset is chosen, the remaining iterations repeatedly measure the signals and hence, suppress the noise of these important patterns. For example, the same number of measurements required for a further 5 iterations of the full pattern set can instead be used for 20 iterations of the $25 \%$ set. The compressed sensing scenarios were evaluated with various fractions of the the full pattern set and SNR constraints. Figure 4 shows the image reconstruction when using pattern sets with differing degrees of selectivity, for up to 5120 measurements in different experimental runs. After every iteration, to measure the quality of the images reconstruction we calculated the RMS error of the reconstruction compared to the ground truth image. ${ }^{13}$

In parallel to the pattern set selectivity, we introduced SNR constraints with 4 scenarios, and characterised the resulting image quality in terms of a root-mean-square error, as shown in figure 5. Of the four cases, the first scenario is that of a near ideal case with a high SNR. No significant advantage is observed to compressed sensing, irrespective of the number of measurements. The best images reconstructed use the full pattern set (Figure $5(\mathbf{A})$ ). Thereafter, three scenarios of increasingly lower SNR were evaluated. It is observed that given a fixed number of measurements, lower RMS errors can be obtained by adopting a compressed sensing approach, repeatedly measuring only the most significant subset of patterns. For instance, we see an improvement in the image quality and lower RMS error after 2048 measurements in scenario $\mathbf{C}$ (Figure 5).

Our result illustrates two key outcomes; (1) that the quality of our patterns formed in the far-field of the modulator allow us to recreate the scene from backscattered light and (2) the method's robustness to facilitate a compressed sensing approach that yields an image improvement in high noise conditions.

\section{CONCLUSIONS}

We have shown a successful implementation of single-pixel imaging using a phase-only modulator to create Hadamard intensity patterns in the far-field of the modulator. We have illustrated the implications of using multiple sources of pattern schemes with the flexibility of application based on specific requirements and/or available equipment. Once the idealised pattern set scheme was chosen, they are used as input along with the signal measurements from the PMT to reconstruct the scene. We also reaffirm the advantages of compressed sensing in constrained conditions with far-field imaging capabilities.

The data underlying the results presented in this paper (i.e. PMT signals, idealised Hadamard patterns) are accessible in the Enlighten (University of Glasgow) repository. ${ }^{23}$

\section{ACKNOWLEDGMENTS}

We wish to acknowledge the support from Leonardo ITG, H2020 Q-Sort (Project ID: 766970), the Royal Society and QuantIC (EP/M01326X/1).

\section{REFERENCES}

[1] Duarte, M. F., Davenport, M. A., Takhar, D., Laska, J. N., Sun, T., Kelly, K. F., and Baraniuk, R. G., "Single-pixel imaging via compressive sampling," IEEE Signal Processing Magazine 25(2), 83-91 (2008).

[2] Gibson, G. M., Johnson, S. D., and Padgett, M. J., "Single-pixel imaging 12 years on: a review," Optics Express 28(19), 28190-28208 (2020).

[3] Howland, G. A., Dixon, P. B., and Howell, J. C., "Photon-counting compressive sensing laser radar for 3D imaging," Applied Optics 50, 5917-5920 (nov 2011). 
[4] Howland, G. A., Lum, D. J., Ware, M. R., and Howell, J. C., "Photon counting compressive depth mapping," Optics Express 21, 23822-23837 (oct 2013).

[5] Sun, M.-J., Edgar, M. P., Gibson, G. M., Sun, B., Radwell, N., Lamb, R., and Padgett, M. J., "Single-pixel threedimensional imaging with time-based depth resolution," Nature Communications 7, 12010 - 6 (07 2016).

[6] Field, J. J., Wernsing, K. A., Domingue, S. R., Motz, A. M. A., DeLuca, K. F., Levi, D. H., DeLuca, J. G., Young, M. D., Squier, J. A., and Bartels, R. A., "Superresolved multiphoton microscopy with spatial frequency-modulated imaging," Proceedings of the National Academy of Sciences of the United States of America 113(24), 6605-6610 (2016).

[7] Dumas, J. P., Lodhi, M. A., Bajwa, W. U., and Pierce, M. C., "From modeling to hardware: an experimental evaluation of image plane and Fourier plane coded compressive optical imaging," Optics Express 25, 29472 - 29491 (2017).

[8] Scotté, C., Sivankutty, S., Stockton, P., Bartels, R. A., and Rigneault, H., "Compressive Raman imaging with spatial frequency modulated illumination," Optics Letters 44(8), 1936-1939 (2019).

[9] Heuke, S., Sivankutty, S., Scotte, C., Stockton, P., Bartels, R. A., Sentenac, A., and Rigneault, H., "Spatial frequency modulated imaging in coherent anti-Stokes Raman microscopy," Optica 7(5), 417-424 (2020).

[10] Studer, V., Bobin, J., Chahid, M., Mousavi, H. S., Candes, E., and Dahan, M., "Compressive fluorescence microscopy for biological and hyperspectral imaging," Proceedings of the National Academy of Sciences of the United States of America 109, 1679-1687 (jun 2012).

[11] Shi, X., Huang, X., Nan, S., Li, H., Bai, Y., and Fu, X., "Image quality enhancement in low-light-level ghost imaging using modified compressive sensing method," Laser Physics Letters 15(4) (2018).

[12] Li, C., Tan, X., Chen, S., and Zhuang, Z., "Optimal ordering strategy of Hadamard measurement basis for single-pixel imaging," in [Proceedings in SPIE 11763, Seventh Symposium on Novel Photoelectronic Detection Technology and Applications ], Su, J., Chu, J., Yu, Q., and Jiang, H., eds., (March), 1-7, SPIE, Kunming, China (2021).

[13] Kallepalli, A., Innes, J., and Padgett, M. J., "Compressed sensing in the far-field of the spatial light modulator in high noise conditions," Scientific Reports 11, 1-8 (8 2021).

[14] Shapiro, J. H., "Computational ghost imaging," Physical Review A - Atomic, Molecular, and Optical Physics 78(6), $1-4$ (2008).

[15] Pratt, W. K., Kane, J., and Andrews, H. C., "Hadamard Transform Image Coding," Proceedings of the IEEE 57(1), 58-68 (1969).

[16] Sloane, N. J. A. and Harwit, M., "Masks for Hadamard transform optics, and weighing designs," Applied Optics 15(1), 107-114 (1976).

[17] Zhang, Z., Wang, X., Zheng, G., and Zhong, J., "Hadamard single-pixel imaging versus Fourier single-pixel imaging," Optics Express 25, 19619-19639 (aug 2017).

[18] Gerchberg, R. W. and Saxton, W. O., "A practical algorithm for the determination of the phase from image and diffraction plane pictures," Optik 35(2) (1972).

[19] Ferri, F., Magatti, D., Lugiato, L. A., and Gatti, A., "Differential ghost imaging," Physical Review Letters 104(25), 1-4 (2010).

[20] Yu, W.-K., Liu, X.-F., Yao, X.-R., Wang, C., Zhai, Y., and Zhai, G.-J., "Complementary compressive imaging for the telescopic system," Scientific Reports 4, 5834 1-6 (jul 2014).

[21] Radwell, N., Mitchell, K. J., Gibson, G. M., Edgar, M. P., Bowman, R., and Padgett, M. J., "Single-pixel infrared and visible microscope," Optica 1, 285-289 (nov 2014).

[22] Denk, O., Musiienko, A., and Ž́́dek, K., "Differential single-pixel camera enabling low-cost microscopy in nearinfrared spectral region," Optics Express 27, 4562-4571 (feb 2019).

[23] Kallepalli, A., Padgett, M. J., and Innes, J., "Single-pixel imaging and compressed sensing in the far-field of the spatial light modulator (Dataset, DOI: 10.5525/gla.researchdata.1133)," (2021). 